\title{
Plate-reader Artifact
}

National Cancer Institute

\section{Source}

National Cancer Institute. Plate-reader Artifact. NCI Thesaurus. Code C87040.

Artifacts resulting from the electronics of the plate reader or from issues associated with the image plate. 